\title{
Reversion of ventricular tachycardia by pacemaker stimulation
}

\author{
M. A. Bennett and B. L. Pentecost \\ From the General Hospital, Birmingham 4
}

\begin{abstract}
Reversion of ventricular tachycardia to either sinus rhythm or atrial fibrillation has been achieved in a group of patients by rapid ventricular pacing. The standard technique involved pacing at a rate slightly in excess of the existing tachycardia until ventricular capture was achieved. Cessation of pacing after a few seconds was usually followed by a supraventricular rhythm, though pacing at a lower rate might be required to suppress completely ventricular ectopic activity. In the event of a return to ventricular tachycardia, ventricular capture was again achieved and the pacing rate gradually slowed to the point where ventricular premature beats first appeared; the heart was then paced slightly in excess of this critical rate.
\end{abstract}

Rapid transvenous pacing of the heart is of value in stabilizing the cardiac rhythm when recurrent episodes of ventricular tachycardia and fibrillation persist in spite of conventional drug treatment (Swedberg and Malm, 1964; Sowton, Leatham, and Carson, 1964; Friedberg, Lyon, and Donoso, 1970; Bennett and Pentecost, 1970). Ventricular tachycardia may, however, return after cessation of pacing or even during the manoeuvre. It seems probable that ventricular tachycardia could be terminated by pacemaker stimulation in a similar way to that described in paroxysmal atrial tachycardia, junctional tachycardia, and atrial flutter (Haft et al., r967; Lister et al., 1968; Hunt et al., 1968. Ventricular tachycardia which progresses rapidly to fibrillation does not offer sufficient time to test this hypothesis but the opportunity does arise when a pacemaker catheter is already in situ after a previous period of prophylactic pacing. This paper describes our initial experience in the attempted reversion of ventricular tachycardia to supraventricular rhythm in a group of patients by means of transvenous pacing of the right ventricle.

\section{Methods}

All pacing procedures were performed in the Hospital's Coronary Care Unit. A transvenous bipolar catheter and a fixed rate pacemaker were used (United Birmingham Hospitals). The catheter was introduced into a medial arm vein at the antecubital fossa and guided to the apex of the right ventricle under fluoroscopic control within the Unit. The electrocardiogram was continuously recorded on electromagnetic tape and permanent paper records made from the tape using an electro-

Received 8 March 1971. cardiograph (Mingograph 243) at a paper speed of $25 \mathrm{~mm} / \mathrm{sec}$.

The patients in this study had all suffered acute myocardial infarction complicated by recurrent ventricular tachyarrhythmias in spite of antiarrhythmic drug therapy. In the majority of patients prophylactic transvenous right ventricular pacing had been employed in an attempt to suppress further ventricular tachycardia, but this rhythm returned either during or after cessation of pacing. It was during a recurrence of ventricular tachycardia that the technique of conversion by rapid pacing was tried. In two further patients the pacing technique was attempted during the first episode of ventricular tachycardia after arrival in the Unit, as described in the relevant case histories.

Reversion of ventricular tachycardia was begun by capturing the ventricle by pacing at a rate slightly in excess of the existing rhythm, with an exception when a slower rate was found to be effective. When the initial rate of pacemaker stimulation failed to capture the ventricle the rate was gradually increased until capture was achieved. Pacing rates in excess of $300 / \mathrm{min}$ were not used. After successful capture, pacing was discontinued abruptly after a few seconds. If supraventricular rhythm was restored no further pacing was performed. In the event of a return to ventricular tachycardia pacing was begun again at the previous capture rate; the paced rate was then gradually reduced until the appearance of ventricular premature beats was observed. The heart was subsequently paced at a rate marginally above this level as a prophylactic measure against further episodes of ventricular tachycardia.

\section{Results}

Case I A 62-year-old woman was admitted with an extensive anterior myocardial infarction. She experienced 12 episodes of ventricular tachyarrhythmia which required DC 
shock. These occurred over a period of 36 hours during which time she received a total of $2.4 \mathrm{~g}$ intravenous lignocaine and $1.5 \mathrm{~g}$ quinidine as well as $500 \mathrm{mg}$ procainamide during the final 8 hours. The cardiac rhythm was satisfactorily stabilized for 48 hours by transvenous pacing but upon cessation of pacing ventricular tachycardia returned.

Pacing was reinstituted at 3 volts and 192 beats/min (Fig. I). This rate was 6 beats/min faster than the existing ventricular tachycardia. Ventricular capture occurred after 3.68 seconds, pacing was continued for a further 3.68 seconds, and then stopped. Two sinus beats followed, the first associated with a ventricular premature beat and the second immediately followed by ventricular tachycardia initiated by a similar premature beat. Pacing was restarted at 3 volts and 192 beats/min. Ventricular capture was immediate (Fig. 2). The pacing rate was reduced in approximately 2 minutes to $\mathrm{I} 38$ beats $/ \mathrm{min}$ and over the following 4 hours to $9 \mathrm{I}$ beats/ min. Pacing was continued at $9 \mathrm{I}$ beats $/ \mathrm{min}$ for 5 days, after which sinus rhythm without ectopic activity was established.

Case 2 This 47-year-old man had been paced after II episodes of cardiac arrest necessitating DC countershock over the course of 2 hours. These episodes had occurred in spite of the administration of a total of $300 \mathrm{mg}$ lignocaine and $250 \mathrm{mg}$ procainamide. The paced rhythm remained stable for 48 hours but ventricular tachycardia returned within a short period of discontinuing pacing.

The catheter had been left in situ and pacing was restarted at 3 volts and a rate of 129 beats/min; this was considerably slower than the ventricular tachycardia of 300 beats/ min (Fig. 3). Ventricular capture was achieved in 1.84 seconds. Pacing at 129 beats/min was continued for approximately 3 minutes and then stopped. Sinus rhythm with ectopic ventricular beats followed. The ectopic beats were abolished by ventricular pacing at II5 beats $/ \mathrm{min}$. Pacing at this rate was maintained for 5 days without recurrence of ventricular tachycardia. Uncomplicated sinus rhythm followed the cessation of pacing.

Case 3 After suffering an inferior infarction this 47-year-old man developed 14 episodes of ventricular tachycardia over the first 2 hours of admission in spite of intravenous lignocaine $(660 \mathrm{mg}$ ) and procainamide $(250$ $\mathrm{mg})$. Though pacing produced a stable rhythm for a short period, 6 further episodes of ventricular tachycardia occurred, the first 4 of which were treated with DC shock. The fifth was managed by rapid pacing at 270 beats/min and ventricular capture was achieved in $\mathrm{I} \cdot \mathrm{I}$ seconds. Pacing at 270 beats $/ \mathrm{min}$ was continued for $2 \cdot 2$ seconds and then stopped. Sinus rhythm with ventricular premature beats followed. A pacing rate of I 50 beats/min was needed to suppress the ventricular ectopic beats. However, after approximately 15 hours ventricular tachycardia recurred at a rate of 270 beats $/ \mathrm{min}$. Rapid ventricular pacing at 275 beats/min resulted in fusion beats but failed to capture the ventricle. The pacemaker rate was increased to 284 beats/min and ventricular capture was achieved. Pacing was continued at 284 beats/min for 2.42 seconds and then stopped. Ventricular tachycardia at 270 beats/min immediately recurred without preceding sinus beats. Pacing was again initiated but at a rate of 300 beats/min; it was continued for 2 seconds and then stopped. Sinus rhythm with ventricular premature beats followed. Prophylactic pacing at 150 beats/min was again required to suppress premature beats in spite of concurrent administration of intravenous lignocaine. The patient developed ventricular fibrillation 3 days later, while being paced, and died.

FIG. I Case I. Ventricular tachycardia in lead VI. Pacing was begun at 'on' and ventricular capture (c) achieved with a pacing rate of 192 beats/min after $3.68 \mathrm{sec}$. On cessation of pacing (off) sinus rhythm returned with coupled ventricular premature beats leading into the original ventricular tachycardia after only two sinus beats.

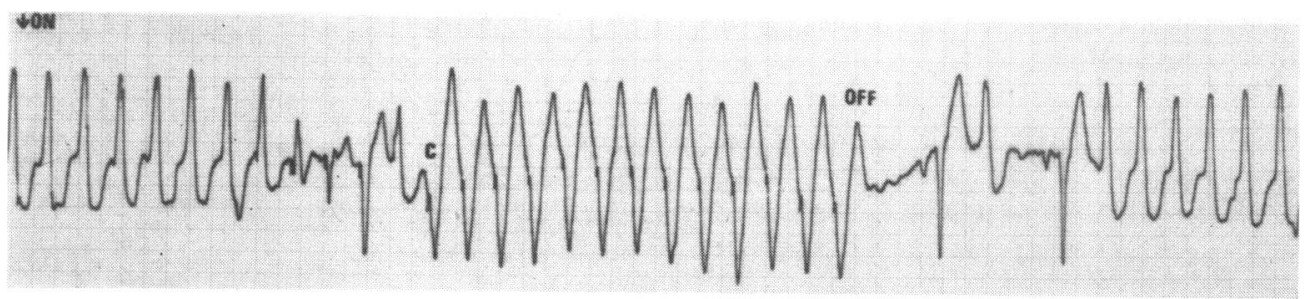



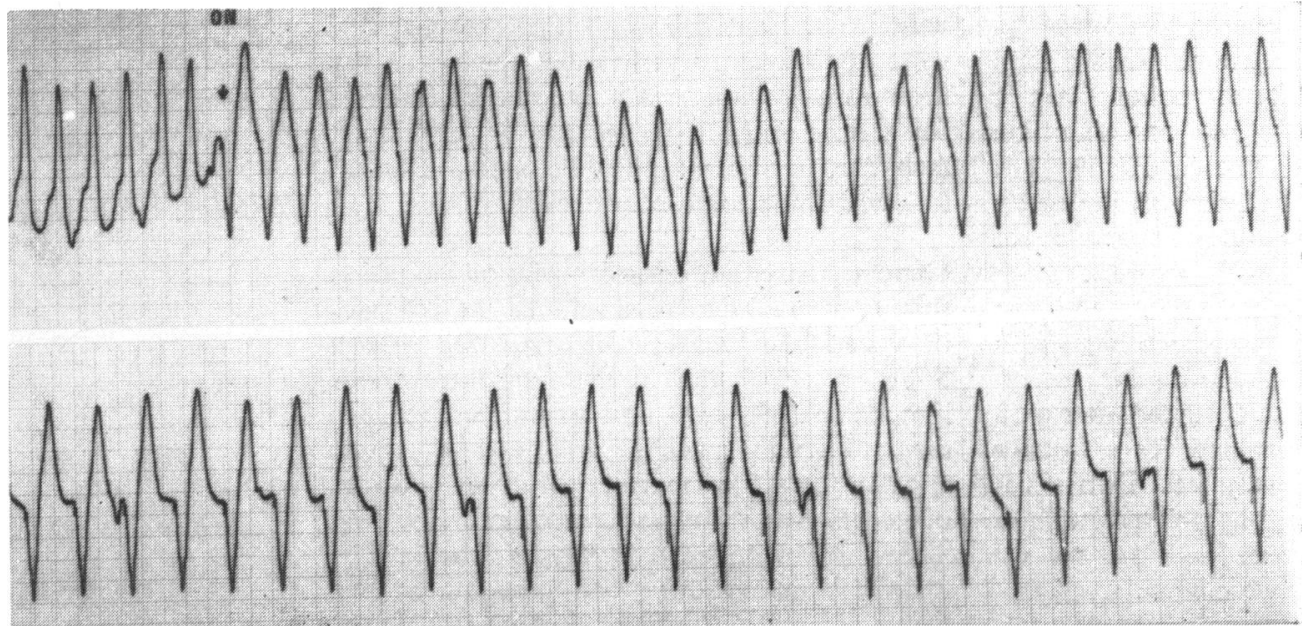

FIG. 2 Case I. Pacing restarted (on) at 192 beats/min with immediate ventricular capture. Lower trace - pacing rate slowed to approximately 138 beats/min with no return of ventricular premature beats.

Case 4 This patient was already in ventricular tachycardia on admission to the Coronary Care Unit. He was a 65-year-old man with an acute anterior infarction. Nineteen episodes of ventricular tachycardia had been treated with DC shock during his short sojourn in the hospital's Accident and Emergency Service, in spite of lignocaine $480 \mathrm{mg}$ which had been administered over approximately 30 minutes. In view of the recurrent nature of the dysrhythmia a pacemaker catheter was introduced into the right ventricle and pacing begun at 2 volts and 250 beats $/ \mathrm{min}$. This was 34 beats/min faster than the intrinsic ventricular tachycardia (Fig. 4). Ventricular capture was achieved after 4.08 sec. Pacing at 250 beats/min was continued for approximately II seconds and then stopped. Sinus rhythm was re-established; ventricular premature beats appeared within minutes and were suppressed by ventricular pacing at 145 beats $/ \mathrm{min}$. Pacing in its third day was intermittent due to instability of the catheter in the right ventricle and during a period of spontaneous sinus rhythm ventricular fibrillation developed which proved fatal.

Case 5 The clinical picture here was similar to that of the previous patient. A 57year-old man with a recent anterior infarction had received DC shock on 17 occasions during I hour in the Casualty Department. The frequency of ventricular tachycardia deteriorating into fibrillation had not been influenced by the intravenous administration of lignocaine $600 \mathrm{mg}$. Between episodes of ventricular tachycardia the underlying rhythm was seen to be atrial fibrillation. A pacemaker catheter was inserted and during an episode of ventricular tachycardia pacing was begun at 3 volts and 215 beats/min, a rate of 29 beats/ min faster than the existing ventricular

FIG. 3 Case 2. Ventricular tachycardia of approximately 300 beats/min. Pacing at I29 beats/min (on) resulted in ventricular capture (c) after $I .84 \mathrm{sec}$.

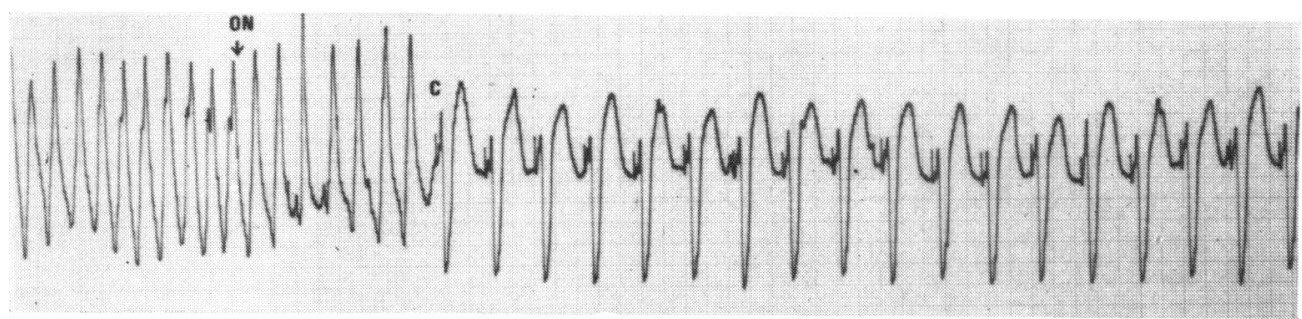




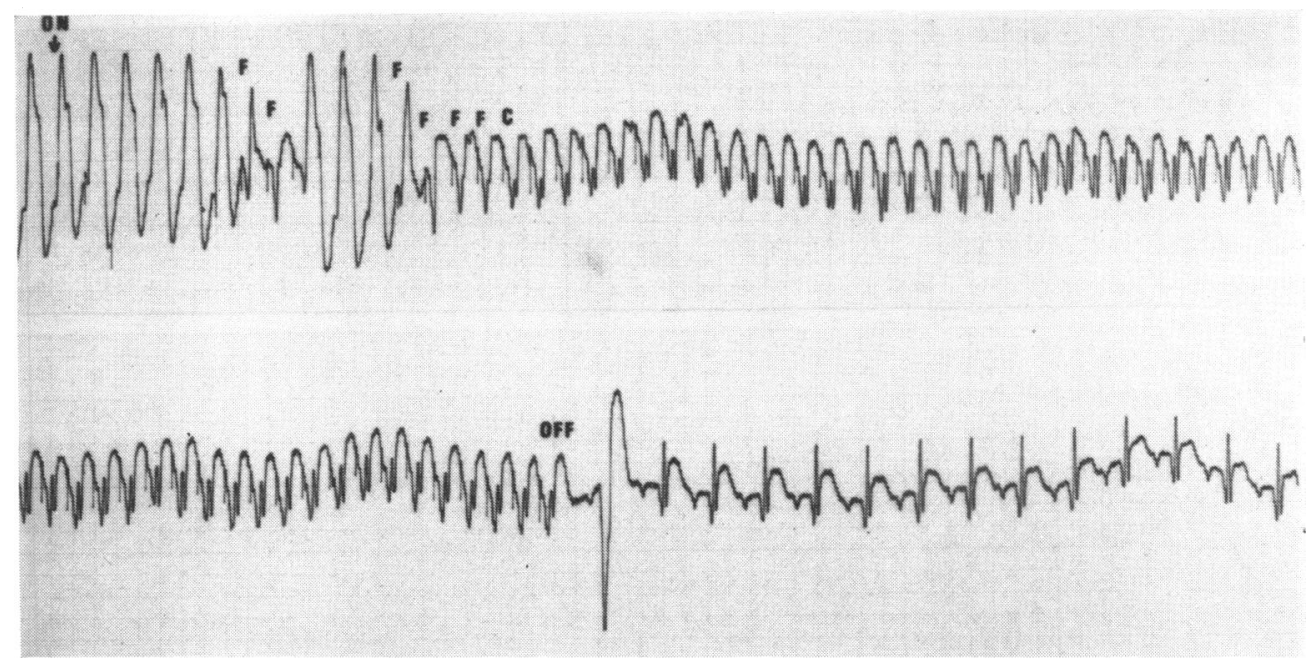

FIG. 4 Case 4. Ventricular tachycardia in lead VI. Pacing begun at 250 beats/min (on), several fusion beats $(F)$ follow, with eventual ventricular capture (c) achieved after $4.08 \mathrm{sec}$. After cessation of pacing (off) there is a return of sinus rhythm - the first sinus beat may have been conducted aberrantly.

tachycardia (Fig. 5). Ventricular capture was achieved after $\mathrm{I} \cdot 68$ seconds. Pacing was stopped after two capture beats. Supraventricular initiated beats followed, but after four such beats ventricular tachycardia recurred. Pacing was restarted at 215 beats/ min. The ventricle was again captured after I.68 seconds (Fig. 6). After ventricular capture the rate was rapidly reduced over two minutes to 138 beats/min and then stopped. Atrial fibrillation followed without interruption.

\section{Discussion}

In four patients return to supraventricular rhythm was achieved by pacing at a rate faster than that of the intrinsic tachycardia.
Reversion in Cases $1,3,4$, and 5 followed pacing at the respective ventricular capture rates for a short period. Case 3, however, on one occasion required a rate in excess of the ventricular capture rate before suppression of the ectopic focus occurred. Ventricular stimulation at a rate in excess of the existing ventricular tachycardia may have permitted return to supraventricular rhythm by suppression of the ectopic ventricular focus by a faster, in this instance artificial, pacemaker - a phenomenon knovin as overdrive suppression (Lange, 1965). The fact that pacing at a rate of 284 beats was sufficient to capture the ventricles but failed to achieve return to supraventricular rhythm on one occasion in Case 3 may have been related to a critical ratedependent entry block to the ectopic focus, an

FIG. 5 Case 5. Ventricular tachycardia in lead VI. Ventricular capture rapidly achieved after pacing was begun (on). After cessation of pacing (off) there were 4 supraventricular initiated beats then a return of the original ventricular tachycardia.

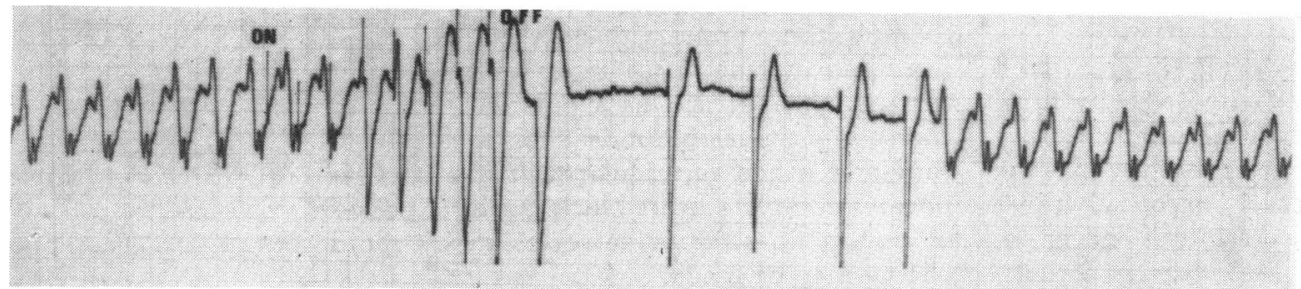




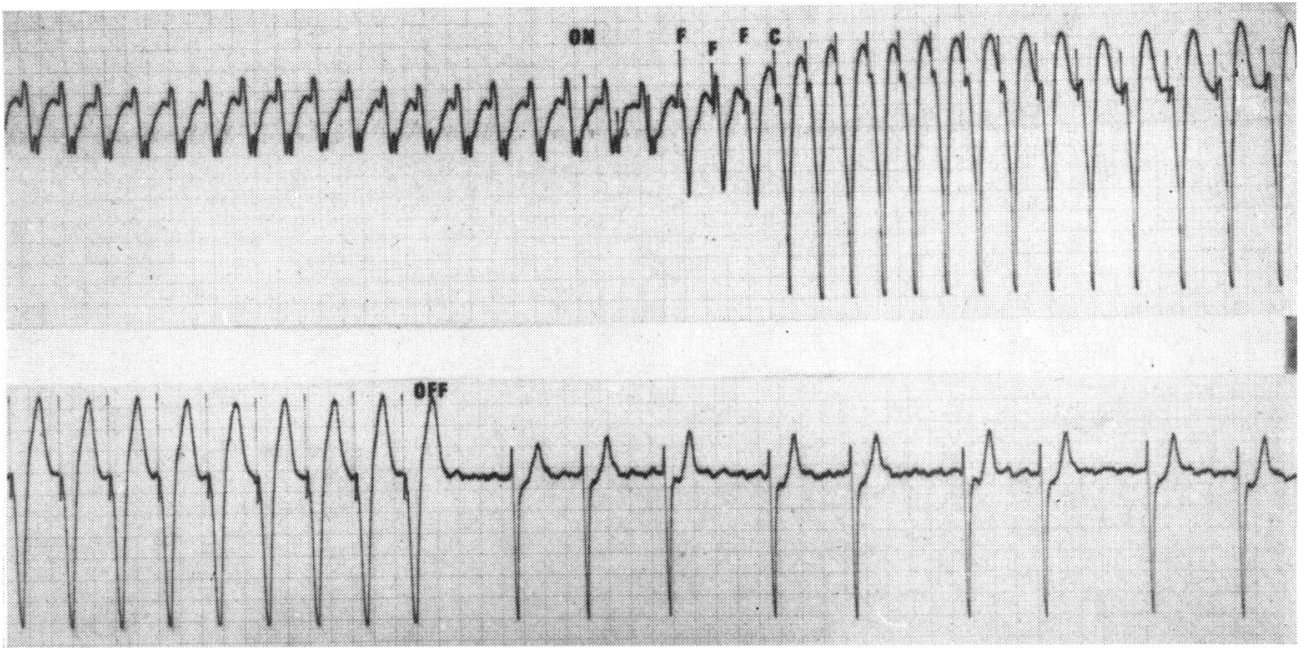

FIG. 6 Case 5. Pacing restarted and ventricular capture (C) achieved after several fusion beats $(F)$. Lower trace: after two minutes the rate of pacing was reduced to 138 beats/min and then stopped. Atrial fibrillation followed without ventricular premature beats.

impediment overcome at a paced rate of 300 beats/min.

Reversion may alternatively have been due to interruption by ventricular paced beats of a re-entry circuit capable of generating tachycardia. Local re-entry circuits may exist after acute infarction due to temporal disparity of repolarization in the peripheral Purkinje tissues (Hoffman and Cranefield, 1964). Moe and colleagues postulated a circuit theory to explain the termination of experimental atrial tachycardia by two electrically induced premature beats (Moe, Cohen, and Vick, 1963). In man, critically timed atrial premature beats have been noted to terminate reciprocating atrial tachycardia (Massumi, Kistin, and Tawakkol, 1967).

The paced rate in Case 2 was less than that of the ventricular tachycardia yet ventricular capture was achieved and sinus rhythm established. The interruption of a circus movement by a suitably timed stimulus may have terminated the rhythm. However, depolarization of the ectopic focus by a pacemaker stimulus occurring in a non-refractory phase of the ventricular tachycardia may have been sufficient to abolish the abnormal focus.

It is worth noting that during the attempted reversion of ventricular tachycardia by fixed rate pacing, ventricular fibrillation was not encountered, possibly due to the fact that the pacemaker stimulus never exceeded three volts and the artificial rate was never in excess of 300 beats/min.
All patients had a history of recurrent ventricular tachyarrhythmias resistant to antiarrhythmic chemotherapy. The majority required pacing after the initial return to sinus rhythm in order to suppress ventricular premature beats. We have found that pacing in this way is effective in the suppression of recurrent ventricular tachyarrhythmias (Bennett and Pentecost, 1970), and the ability to correct or control recurrent ventricular tachycardia developing either during or after pacing is a useful extension of the technique. It does not require general anaesthesia and avoids the hazards of high energy electrical cardioversion (Lown, Amarasingham, and Neuman, 1962), an important consideration when recurrent ventricular tachycardia requires repeated cardioversion in a patient with an already damaged myocardium. Introduction of a pacemaker catheter requires time, however, and the sudden onset of ventricular tachycardia should usually be treated by the immediate injection of antiarrhythmic drugs and preparation for DC shock under general anaesthesia if necessary. In those patients, however, in whom the episodes of tachyarrythmia occur frequently, in spite of drug therapy, there appears to be a case for rapid fixed rate pacing, both in order to prevent further episodes and as a possible means of correcting the arrhythmia should it return. Similarly, when ventricular tachycardia complicates complete heart block during a period of pacing an attempt at reversion by rapid pacing may be made. 


\section{References}

Bennett, M. A., and Pentecost, B. L. (1970). The suppression of ventricular tachyarrhythmias by transvenous intracardiac pacing after acute myocardial infarction. British Medical fournal, 4, 468.

Friedberg, C. K., Lyon, L. J., and Donoso, E. (1970). Suppression of refractory recurrent ventricular tachycardia by transvenous rapid cardiac pacing and antiarrhythmic drugs. Report of seven cases. American Heart fournal, 79, 44.

Haft, J. I., Kosowsky, B. D., Lau, S. H., Stein, E., and Damato, A. N. (1967). Termination of atrial flutter by rapid electrical pacing of the atrium. American fournal of Cardiology, 20, 239.

Hoffman, B. F., and Cranefield, P. F. (1964). The physiological basis of cardiac arrhythmias. American Fournal of Medicine, 37, 670.

Hunt, N. C., Cobb, F. R., Waxman, M. B., Zeft, H. J., Peter, R. H., and Morris, J. J. (1968). Conversion of supraventricular tachycardias with atrial stimulation. Evidence for re-entry mechanisms. Circulation, 38, 1060.

Lange, G. (1965). Action of driving stimuli from intrinsic and extrinsic sources on in situ cardiac pacemaker tissues. Circulation Research, 17, 449.
Lister, J. W., Cohen, L. S., Bernstein, W. H., and Samet, P. (1968). Treatment of supraventricular tachycardias by rapid atrial stimulation. Circulation, 38, 1044 .

Lown, B., Amarasingham, R., and Neuman, J. (1962). New method for terminating cardiac arrhythmias; use of synchronized capacitor discharge. Fournal of the American Medical Association, 182, 548.

Massumi, R. A., Kistin, A. D., and Tawakkol, A. A. (1967). Termination of reciprocating tachycardia by atrial stimulation. Circulation, 36, 637.

Moe, G. K., Cohen, W., and Vick, R. L. (1963). Experimentally induced paroxysmal A-V nodal tachycardia in the dog: a 'case report'. American Heart Fournal, $65,87$.

Sowton, E., Leatham, A., and Carson, P. (1964). The suppression of arrhythmias by artificial pacemaking. Lancet, 2, 1098.

Swedberg, J., and Malm, A. (1964). Pacemaker stimulation in ventricular paroxysmal tachycardia. Acta Chirurgica Scandinavica, 128, 6 ro.

Requests for reprints to Dr. B. L. Pentecost, The General Hospital, Steelhouse Lane, Birmingham, B4 6NH. 